\title{
RESEARCH
}

\section{Heterogeneity of genomic profile in patients with HER2-positive breast cancer}

\author{
Bo Chen1,*, Guochun Zhang1,*, Guangnan Wei1,*, Yulei Wang1,2, Liping Guo1,3, Jiali Lin1,3, Kai Li1, Hsiaopei Mok',

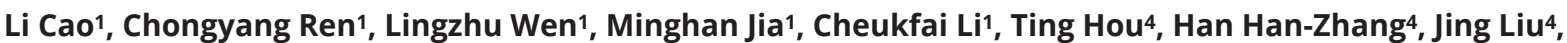 \\ Charles M Balch ${ }^{5}$ and Ning Liao' ${ }^{1}$
}

1Department of Breast Cancer, Cancer Center, Guangdong Provincial People's Hospital and Guangdong Academy of Medical Sciences, Guangzhou, Guangdong, China

2School of Medicine, South China University of Technology, Guangzhou, Guangdong, China

${ }^{3}$ The Second School of Clinical Medicine, Southern Medical University, Guangzhou, Guangdong, China

${ }^{4}$ Burning Rock Biotech, Guangzhou, Guangdong, China

${ }^{5}$ Department of Surgical Oncology, The University of Texas MD Anderson Cancer Center, Houston, Texas, USA

Correspondence should be addressed to N Liao: syliaoning@scut.edu.cn

*(B Chen, G Zhang and G Wei contributed equally to this work)

\begin{abstract}
HER2-positive breast cancer is a biologically and clinically heterogeneous disease. Based on the expression of hormone receptors (HR), breast tumors can be further categorized into HR positive and HR negative. Here, we elucidated the comprehensive somatic mutation profile of HR+ and HR- HER2-positive breast tumors to understand their molecular heterogeneity. In this study, 64 HR+/HER2+ and 43 HR-/HER2+ stage I-III breast cancer patients were included. Capture-based targeted sequencing was performed using a panel consisting of 520 cancer-related genes, spanning 1.64 megabases of the human genome. A total of 1119 mutations were detected among the 107 HER2-positive patients. TP53, CDK12 and PIK3CA were the most frequently mutated, with mutation rates of 76, 61 and 49, respectively. HR+/HER2+ tumors had more gene amplification, splice site and frameshift mutations and a smaller number of missense, nonsense and insertiondeletion mutations than HR-/HER2+ tumors. In KEGG analysis, HR+/HER2+ tumors had more mutations in genes involved in homologous recombination $(P=0.004)$, TGF-beta $(P=0.007)$ and WNT $(P=0.002)$ signaling pathways than HR-/HER2+ tumors. Moreover, comparative analysis of our cohort with datasets from The Cancer Genome Atlas and Molecular Taxonomy of Breast Cancer International Consortium revealed the distinct somatic mutation profile of Chinese HER2-positive breast cancer patients. Our study revealed the heterogeneity of somatic mutations between HR+/HER2+ and HR-/HER2+ in Chinese breast cancer patients. The distinct mutation profile and related pathways are potentially relevant in the development of optimal treatment strategies for this subset of patients.
\end{abstract}
Key Words
- HER2-positive breast cancer
- Chinese breast cancer
- hormone receptor
- mutation landscape
- ERBB family 


\section{Introduction}

Breast cancer is one of the most common cancer types with great heterogeneity (Cancer Genome Atlas Network 2012, DeSantis et al. 2017). The genetic and molecular characteristics of the breast tumor could strongly influence treatment response and prognosis (Pitt et al. 2018). Gene amplification of human EGF receptor 2 (HER2, also called ERBB2), located in chromosomal region $17 \mathrm{q} 12$, defines a subset of approximately $15 \%$ of all clinically diagnosed breast cancers (Ferrari et al. 2016). HER2-amplified breast tumors are effectively targeted with agents including monoclonal antibodies and tyrosine kinase inhibitors (Piccart-Gebhart et al. 2016). However, a substantial difference exists in the clinical behavior of HER2-positive breast cancer, such as rate of pathologic complete response (pCR) and pattern of relapse and metastasis. Intratumor genetic heterogeneity has been implicated as the molecular mechanism of this variability in treatment response to targeted therapies (Ng et al. 2015).

Based on the expression of hormone receptors (HR), including estrogen receptor (ER) and progesterone receptor (PR), HER2-positive breast cancers can be further categorized into HR+/HER2+ or HR-/HER2+ subtypes. Previous clinical trials have demonstrated the influence of HR and HER2 status on the differential efficacy of HER2 targeted therapies (Von Minckwitz et al. 2012, Cortazar et al. 2014). Clearly, there is a major need to better understand the intrinsic genomic characteristics of HER2positive tumors to further improve treatment outcomes of HER2-positive patients (Gingras et al. 2017). Numerous efforts have been invested to reveal the molecular features and the factors that affect treatment responses. A study reported the association between the presence of PIK3CA mutations and a poorer pCR rate in HER2-positive breast cancer patients who received trastuzumab, lapatinib or a combination of the two (Loibl et al. 2014). Moreover, substantial data suggest that somatic mutations in ERBB2 family of genes, including EGFR, HER2, HER3 and HER4, could induce ligand-independent constitutive HER2 receptor signaling, promote oncogenesis and influence response to HER2-targeted therapies (Jaiswal et al. 2013, Toomey et al. 2017, Hyman et al. 2018). However, the comprehensive genetic characteristics of HER2-positive tumors including the genetic difference between HR+/HER2+ and HR-/HER2+ breast cancer remain uncharacterized.

In this study, we aimed to elucidate the comprehensive somatic mutation landscape of HR+/HER2+ and HR-/HER2+ breast tumors. We utilized capture-based ultra-deep targeted sequencing to interrogate the comprehensive somatic mutation profiles of 64 HR+/HER2+ and 43 HR-/HER2+ stage I-III breast cancer patients using a panel consisting of 520 cancer-related genes, spanning $1.64 \mathrm{Mb}$ of the human genome.

\section{Materials and methods}

\section{Patients}

In this study, we enrolled $64 \mathrm{HR}+/ \mathrm{HER} 2+$ and 43 HR-/HER2+ with various stages (stage I-III), excluding stage IV, from Guangdong Provincial People's Hospital. The patients' clinical characteristics are shown in Supplementary Table 1 (see section on supplementary materials given at the end of this article). ASCO/College of American Pathologists guidelines were used to define ER, PR and HER2 positivity. HR-positive status was defined as ER positivity regardless of PR status, while HR-negative status was defined as having negative status for both ER and PR. Patients in HR+/HER2+ and HR-/HER2+ groups were comparable in age, menopausal status, stage, histological grade and histological type. This study was approved by the Ethics Committee of Guangdong Provincial People's Hospital. Written informed consent was obtained from each patient.

\section{Tissue DNA extraction}

DNA was extracted using QIAamp DNA FFPE tissue kit (Qiagen). DNA concentration was measured using Qubit dsDNA assay.

\section{NGS library preparation and capture-based targeted DNA sequencing}

NGS library preparation and sequence data analysis were conducted according to our previous study (Zhang et al. 2019). Genomic profiling was performed using a panel covering 520 cancer-related genes (OncoScreen Plus, Burning Rock Biotech, Guangzhou, China). Whole exons of 312 genes and critical exons, introns and promoter regions of the remaining 208 genes were captured. Ten milliliters of peripheral blood was obtained. Events were then classified as germline or somatic depending on their presence in the matched normal set (white blood cells) of events.

\section{Sequence data analysis}

Sequence data were mapped to the human genome (hg19) using BWA aligner v.0.7.10. Local alignment optimization, (c) 2020 Society for Endocrinology Published by Bioscientifica Ltd. Printed in Great Britain 
variant calling and annotation were performed using GATK v.3.2 and VarScan v.2.4.3. Variants were filtered using the VarScan fpfilter pipeline, with loci with depth less than 100 filtered out. At least five supporting reads were needed for INDELs, while eight supporting reads were needed for single nucleotide variations (SNVs) to be called. According to the ExAC, 1000 Genomes, dbSNP, and ESP6500SI-V2 databases, variants with population frequency over $0.1 \%$ were grouped as SNP and excluded from further analysis. Remaining variants were annotated with ANNOVAR and SnpEff v.3.6. DNA translocation analysis was performed using Factera v.1.4.3.

\section{Statistical analysis}

Data were summarized by frequency and percentage for categorical variables including mutation detection rate and distribution of mutation types. Comparisons between groups were performed using Fisher's exact test or chi-square test for these categorical variables wherever applicable. Wilcoxon signed-rank test was used for calculating the significance between individual mutation types in HR+ and HR- groups. All statistical tests were two-sided and differences were considered significant when $P$ value was $<0.05$.

\section{Results}

\section{Mutation landscape of HER2-positive breast cancers}

A total of 1119 mutations, including 478 single nucleotide variants (SNVs), 14 insertions or deletions (Indels), 29 fusions, 593 copy number $(\mathrm{CN})$ amplifications, 2 large genomic rearrangements and $3 \mathrm{CN}$ deletions, were detected in 267 genes from 107 patients. As shown in Fig. 1, ERBB2 CN amplifications were detected from 98 tumors, achieving a concordance rate of 91.6\% (98/107) with traditional methods of HER2 assessment. In addition to ERBB2, mutations detected from HER2-positive breast cancers included TP53 (76\%), CDK12 (61\%), PIK3CA (49\%), SPOP (19\%), RARA (18\%), MYC (15\%), CCND1 (12\%), FGF19 (12\%), FGF3 (12\%), FGF4 (12\%), RNF43 (12\%), NBN (12\%), HNF1B (12\%) and BRIP1 (11\%). Among them, TP53, CDK12 and PIK3CA were the most frequently mutated genes from our cohort.

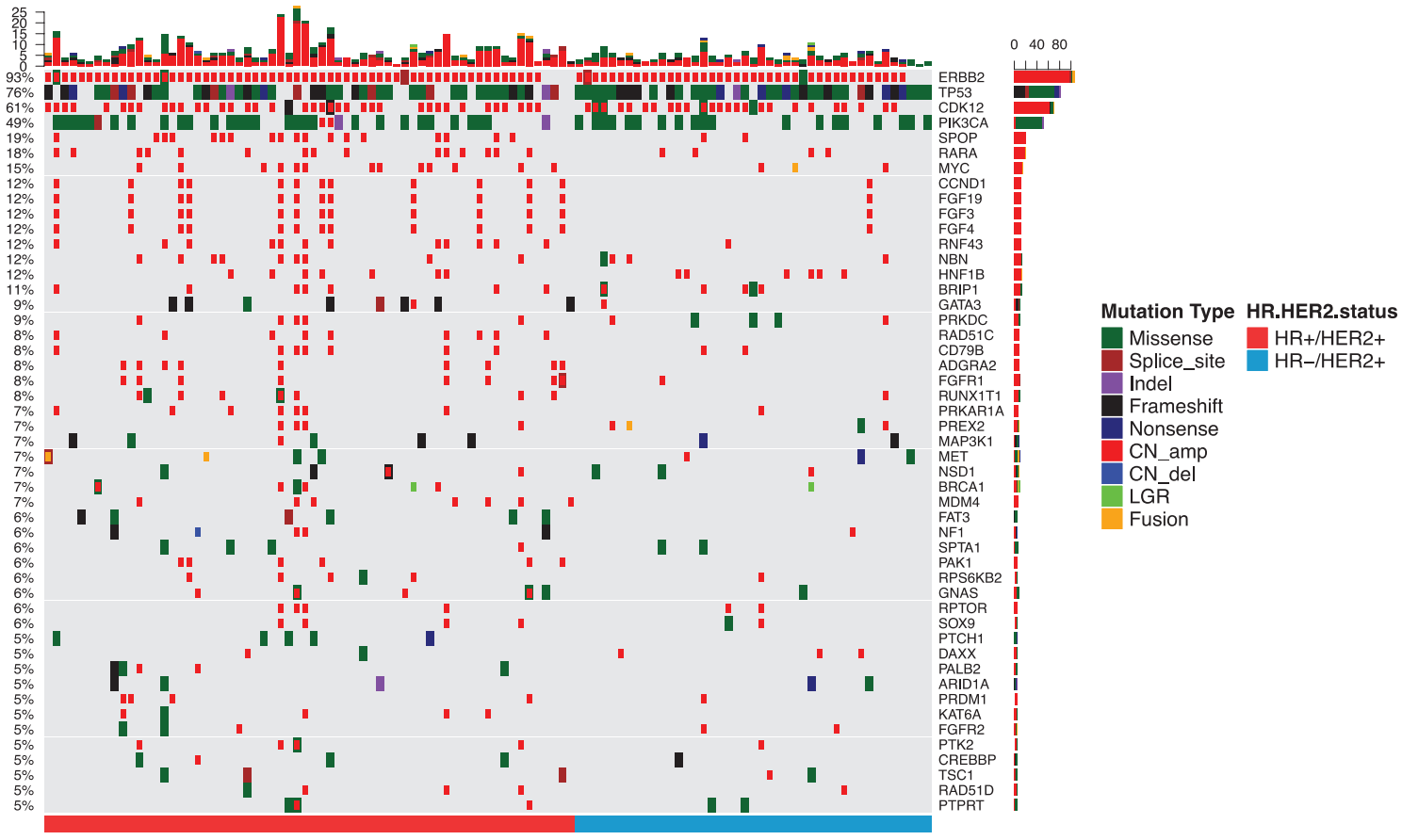

\section{Figure 1}

Summary of genomic features of the 107 HER2-positive breast cancer patients. Genomic alterations of more than $5 \%$ are shown in the Oncoprint. Tumor samples were grouped according to HR status as: HR+/HER2 $+(n=64)$ and HR-/HER2+ $(n=43)$. Top bar summarizes the total number of mutations in each patient (columns); side bar (rows) summarizes the percentage of tumors with mutation in each gene (left) and mutation composition for each gene in the entire cohort (right). Different colors denote different types of mutations and different clinicopathological features. Indel, insertions or deletions; CN_amp, copy number amplification; CN_del, copy number deletion. 


\section{Mutation type distribution and pathway analysis in HR+/HER2+ and HR-/HER2+ patients}

In HER2-positive breast cancers, the HR expression status directly affects the biological behavior of the tumor and affects the treatment and prognosis of the patients. Therefore, we analyzed the distribution of the mutation type and the pathways commonly mutated in tumors with either HR+/HER2+ or HR-/HER2+ that could contribute to the differences observed between the two groups.

Interestingly, tumors with HR+/HER2+ had more somatic mutations than tumors with HR-/HER2+ (767 vs 352 , Table 1). The mutation detection rates were 11.98 and $8.19 \%$ in tumors with HR+/HER2+ and HR-/HER2+, respectively. As summarized in Table 1, the distribution of mutation types was significantly different between HR+/HER2+ and HR-/HER2+ $(P=0.017)$. HR+/HER2+ tumors had more $\mathrm{CN}$ amplification $(P=0.037)$, splice site $(P=0.013)$, and frameshift mutations $(P=0.046)$, a trend of less number of nonsense mutations $(P=0.087)$, and similar number of missense $(P=0.895)$ and indel $(P=1)$ mutations as compared to HR-/HER2+ tumors.

Next, we used the Kyoto Encyclopedia of Genes and Genomes (KEGG) database to analyze the pathways that are distinct between the two groups. Our results demonstrate that the major signaling pathways affected in HR+/HER2+ tumors were homologous recombination (Fig. $2 \mathrm{~A}, 37.5 \%$ vs $11.6 \%, P=0.004$ ), TGF-beta (Fig. $2 \mathrm{~B}, 35.9 \%$ vs $11.6 \%, P=0.007$ ) and WNT (Fig. $2 \mathrm{C}, 45.3 \%$ vs $16.3 \%$, $P=0.002)$. Genes involved in HRR pathway detected from our cohort included $B L M, B R C A 2, N B N, P O L D 1$, $R A D 51 B / C / D$ and RAD52. Among them, NBN $(n=4)$ and RAD51D $(n=1)$ were the only mutations detected from five patients with HR-/HER2+ tumors. Meanwhile, mutations in NBN $(n=9), R A D 51 C(n=9), R A D 51 D(n=4)$, POLD1 $(n=3), B L M(n=1), B R C A 2(n=1), R A D 51 B(n=1)$ and RAD52 $(n=1)$ were detected in 24 patients with

Table 1 Mutation type distribution of HR+/HER2+ and HR-/HER2+ patients. Bold indicates statistical significance, $P<0.05$.

\begin{tabular}{lcc}
\hline & & HR+/HER2+ $(n=64)$ \\
\cline { 1 - 1 } CN_amp & & $424(55.3 \%)$ \\
CN_del & & $3(0.4 \%)$ \\
Missense & & $227(29.6 \%)$ \\
Frameshift & & $41(5.3 \%)$ \\
Nonsense & & $15(2 \%)$ \\
Splice_site & & $30(3.9 \%)$ \\
Indel & & $7(0.9 \%)$ \\
Fusion & & $19(2.5 \%)$ \\
LGR & $1(0.1 \%)$ \\
Total & 767 \\
\hline
\end{tabular}

\begin{tabular}{ccc}
\hline HR-/HER2+ $(n=43)$ & & P value \\
\cline { 1 - 1 } $169(48.0 \%)$ & & $\mathbf{0 . 0 2 4}$ \\
$0(0.0 \%)$ & & 0.240 \\
$130(36.9 \%)$ & & $\mathbf{0 . 0 1 5}$ \\
$15(4.3 \%)$ & & 0.440 \\
$14(4 \%)$ & & $\mathbf{0 . 0 4 8}$ \\
$6(1.7 \%)$ & & 0.052 \\
$7(2.0 \%)$ & & 0.133 \\
$10(2.8 \%)$ & & 0.722 \\
$1(0.3 \%)$ & 0.572 \\
352 & 0.017 \\
\hline
\end{tabular}

(c) 2020 Society for Endocrinology Published by Bioscientifica Ltd. Printed in Great Britain
HR+/HER2+ tumors. Four patients with HR+/HER2+ tumors had concurrent mutations in at least two genes. Genes involved in the TGF- $\beta$ signaling pathway detected from our cohort included CREBBP, EP300, ID3, INHBA, MAPK3, MYC, RPS6KB2, SMAD2/3/4 and PPP2R1A. Genes involved in the WNT pathway detected from our cohort included APC, AXIN1/2, CCND1, CREBBP, CTNNB1, EP300, MYC, RAC1, SMAD4 and TCF7L2.

Taken together, these data indicate that HR+/HER2+ and HR-/HER2+ tumors are molecularly distinct based on the difference in the number and distribution of somatic mutation types detected in these tumors as well as the major pathways affected by these mutations.

\section{Differentially mutated genes between HR+/HER2+ and HR-/HER2+}

Next, we compared the differentially mutated genes between HR+/HER2+ and HR-/HER2+ tumors. Figure 3 illustrates the ten genes found to be differentially mutated between the two groups. Concurrent $\mathrm{CN}$ amplifications in SPOP, CCND1, FGF19, FGF4, FGF3, RNF43, RAD51C, ADGRA2 and MDM4 and various mutations in GATA3 were more frequent in $\mathrm{HR}+/ \mathrm{HER} 2+$ tumors than HR-/ HER2+ tumors.

\section{Distinct somatic mutation profile of Chinese HER2+ breast cancer patients}

To further understand whether the somatic mutation profile of the Chinese HER2+ breast cancer patients are ethnically distinct, we compared the data from our cohort with the HR+/HER2+ and HR-/HER2+ breast cancer dataset from The Cancer Genome Atlas (TCGA) (Cancer Genome Atlas Network 2012) and Molecular Taxonomy of Breast Cancer International Consortium (METABRIC) (Curtis et al. 2012).

First, we compared the data within the respective dataset. Our analysis revealed that in the TCGA dataset, HR+/HER2+ patients had significantly less mutations in genes involved in the cell cycle pathway (58.3 vs $88.9 \%, P=0.025)$ and TGF- $\beta$ signaling pathway (22.2 vs $55.6 \%, P=0.009)$ than HR-/HER2+ patients. Mutations in genes involved in the cell cycle observed from the TCGA breast cancer cohort included $A B L 1, A T M, A T R$, CCND1/2/3, CCNE1, CDK4, CDKN1B, CHEK1/2, EP300, HDAC2, MDM2, MYC, PRKDC, RB1, SMAD2/3/4, STAG2 and TP53. Meanwhile, genes involved in the TGF- $\beta$ signaling observed from the TCGA breast cancer cohort included ACVR1B, EP300, MYC, RPS6KB2, SMAD2/3/4 


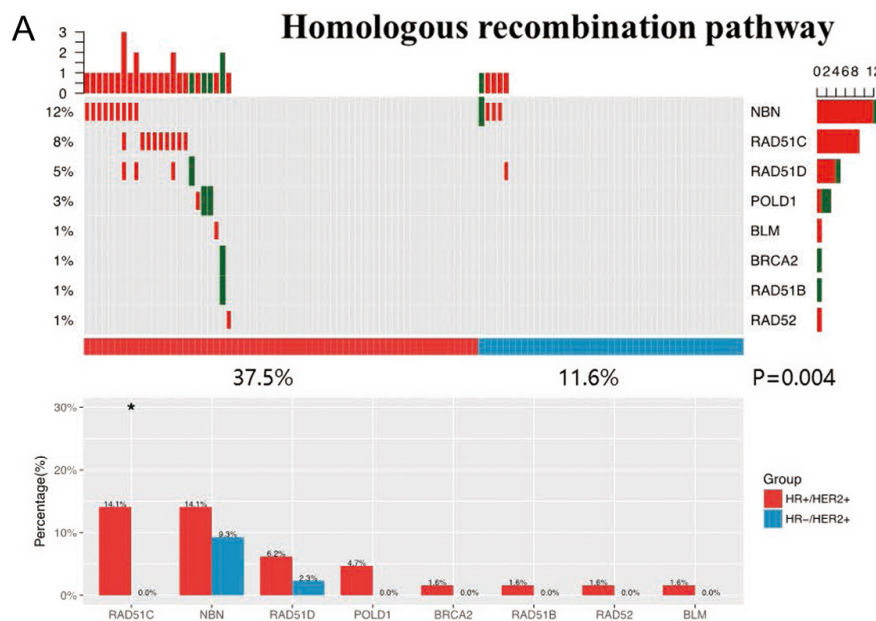

B
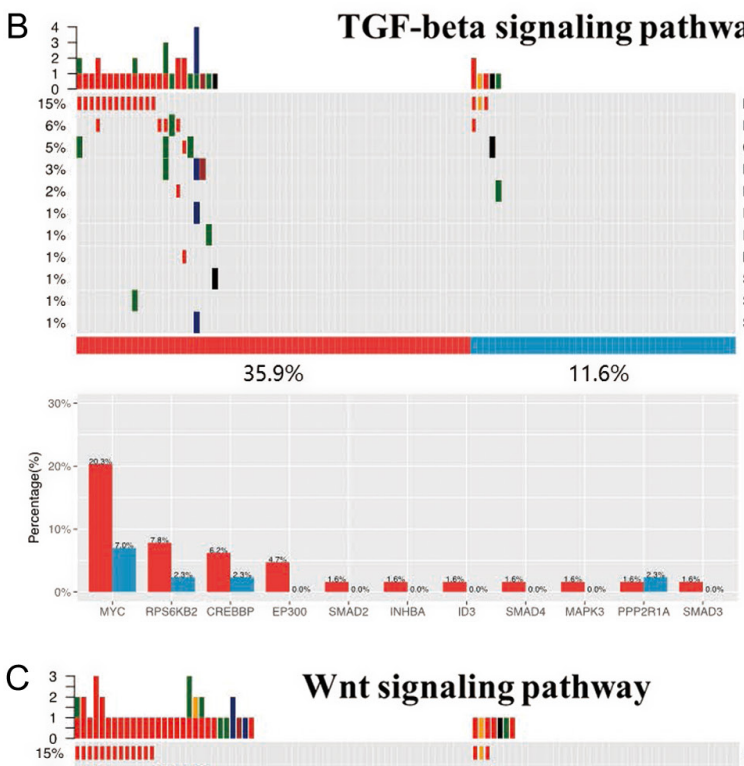

$15 \%$

$12 \%$ I'I

${ }_{3 \%}$ ।

$3 \%$

$3 \%$
$1 \%$
$1 \%$

$1 \%$

$1 \%$
$1 \%$
$1 \%$
$1 \%$

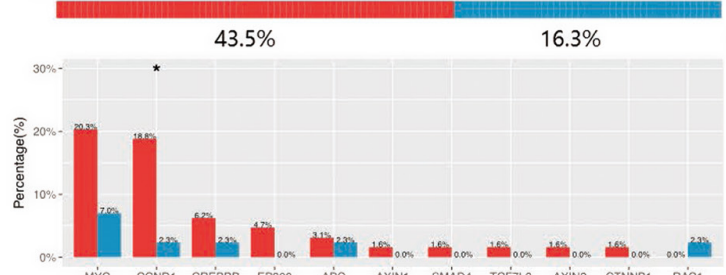

Wnt signaling pathway
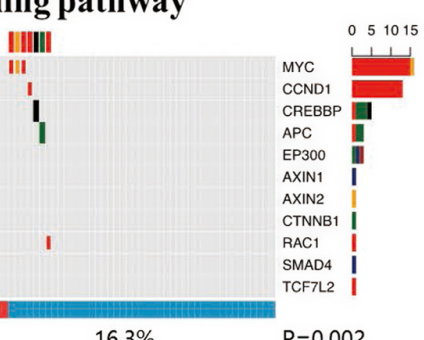

Group
HR+HER2
HR-MER2 and TGFBR1. Meanwhile, in the METABRIC dataset, the cell cycle pathway (77.9 vs $88.1 \%, P=0.039)$ and the PI3K-AKT signaling pathway (90.3 vs $80.6 \%, P=0.048$ ) were significantly different between $\mathrm{HR}+/ \mathrm{HER} 2+$ and HR-/HER2+.

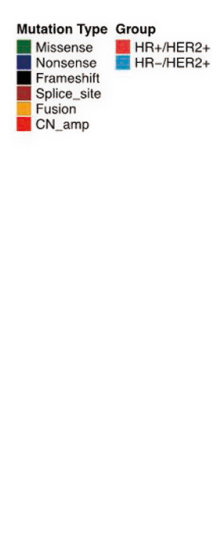

\section{Figure 2}

KEGG analysis reveals distinct pathways in HR+/HER2+ and HR-/HER2+ tumors. The three pathways with the significant enrichments in HR+/HER2+ as compared to HR-/HER2+ samples. (A) Homologous recombination pathway (37.5 vs $11.6 \%, P=0.004) ;(B)$ TGF-beta signaling pathway (35.9\% vs $11.6 \%, P=0.007)$; (C) Wnt signaling pathway (35.9\% vs $11.6 \%, P=0.002)$. Alterations in genes participating in any of the three pathways were listed. Each column represents a sample and each row represents a gene. Mutation types were denoted in different colors. Samples were grouped according to the HR status. The percentage of samples in each group with a specific mutation was shown in the bottom panel.

These analyses suggest that the primary pathways that drive HR+/HER2+ and HR-/HER2+ breast cancer development are significantly different among the datasets. We further compared our cohort and the TCGA and METABRIC datasets in the pathways found to be 


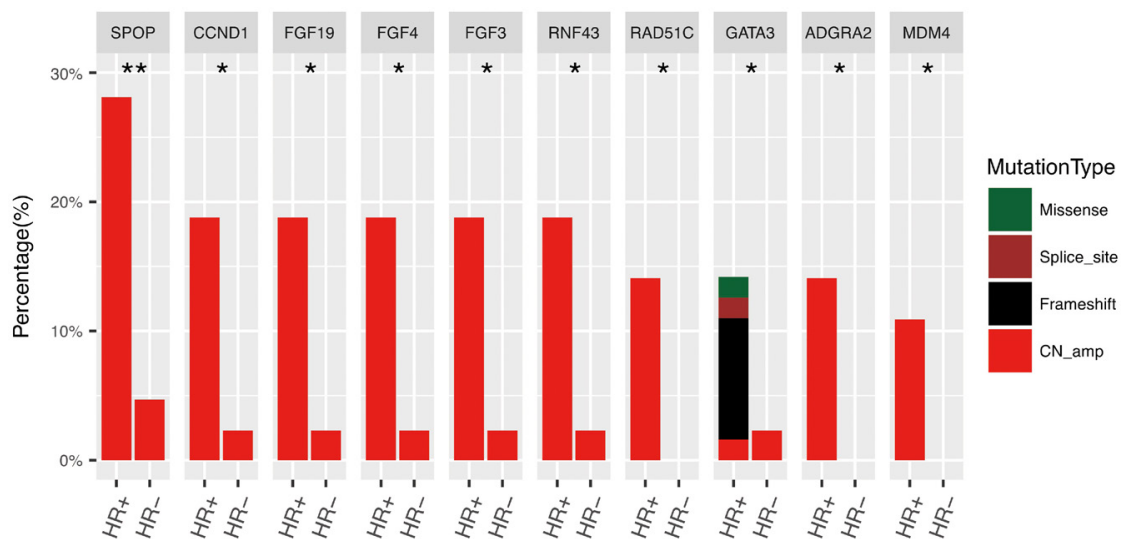

\section{Figure 3}

Differentially mutated genes between HR+/HER2+ and HR-/HER2+ patients. Genes with significantly different mutation frequencies in $\mathrm{HR}+/ \mathrm{HER} 2+$ as compared to HR-/HER2+ samples. Y-axis represents the percentage of samples with mutations in a specific gene. Mutation types were denoted in different colors. * indicated $P$ values $<0.05, * *$ indicated $P$ values $<0.01$. A full colour version of this figure is available at https://doi. org/10.1530/ERC-19-0414. highly mutated among patients with HR+/HER2+ tumors from our cohort. Among the patients with HR+/HER2+ tumors, our cohort was comparable to the TCGA dataset in mutations in genes that are involved in homologous recombination (37.5 vs $29.2 \%, P=0.362)$, TGF- $\beta$ (32.8 vs $22.2 \%, P=0.181)$ and WNT (42.2 vs $36.1 \%, P=0.486)$ signaling pathways. On the contrary, when compared with the HR+/HER2+ patients from the METABRIC dataset, our cohort had a significantly lower frequency of mutations in the homologous recombination (55.8 vs $37.5 \%, P=0.028)$, TGF- $\beta$ (32.8 vs $51.3 \%, P=0.0189)$ and WNT (42.2 vs $54.0 \%, P=0.159)$ signaling pathways.

We further explored whether the distinct mutation type distribution we observed between our patients with HR+/HER2+ and HR-/HER2+ tumors is specific to Chinese patients. We then compared the mutation type distribution in the HR+/HER2+ and HR-/HER2+ tumors from the TCGA and METABRIC cohorts. The mutation type distribution of the HR+/HER2+ and HR-/HER2+ tumors from the TCGA dataset were not statistically different. Meanwhile, the HR+/HER2+ from the METABRIC dataset had significantly more $\mathrm{CN}$ amplification $(P=0.021)$ than the HR-/HER2+ tumors, while the other mutation types were comparable between the HR+/HER2+ and HR-/HER2+ tumors. Further analysis revealed that our $\mathrm{HR}+/ \mathrm{HER} 2+$ cohort had significantly lesser number of $\mathrm{CN}$ amplifications than the METABRIC dataset $(P<0.001)$, but was not statistically different from the TCGA dataset $(P=0.338)$.

In addition to the significantly mutated pathways, we also explored the overall mutation rates of TP53, PIK3CA and CDK12 in HER2+ breast cancer in general (Fig. 4). Analysis revealed significantly higher mutation rates in TP53 (our cohort vs TCGA, 76.6 vs $38.9 \%, P<0.001$; our cohort vs METABRIC, 76.6 vs $66.8 \%, P=0.078)$, PIK3CA (our cohort vs TCGA, 50.5 vs $35.6 \%, P=0.044$; our cohort vs METABRIC, 50.5 vs $35.6 \%, P=0.010)$ and $C D K 12$ (our cohort vs TCGA, 66.4 vs $45.6 \%, P=0.004$ ). Meanwhile, our cohort had significantly lower $C D K 12$ mutation rate than the METABRIC cohort ( 66.4 vs $83.8 \%, P<0.001)$.

Taken together, our data strongly indicate that the Chinese HR+/HER2+ and HR-/HER2+ breast cancer patients have distinct somatic mutation profiles than their Caucasian counterpart.

\section{Concomitant somatic mutations in the ERBB family}

The ERBB family consists of four receptor tyrosine kinases, EGFR (ERBB1), ERBB2 (HER2), ERBB3 (HER3) and ERBB4 (HER4). As shown in Table 2, except for HER2 amplification,

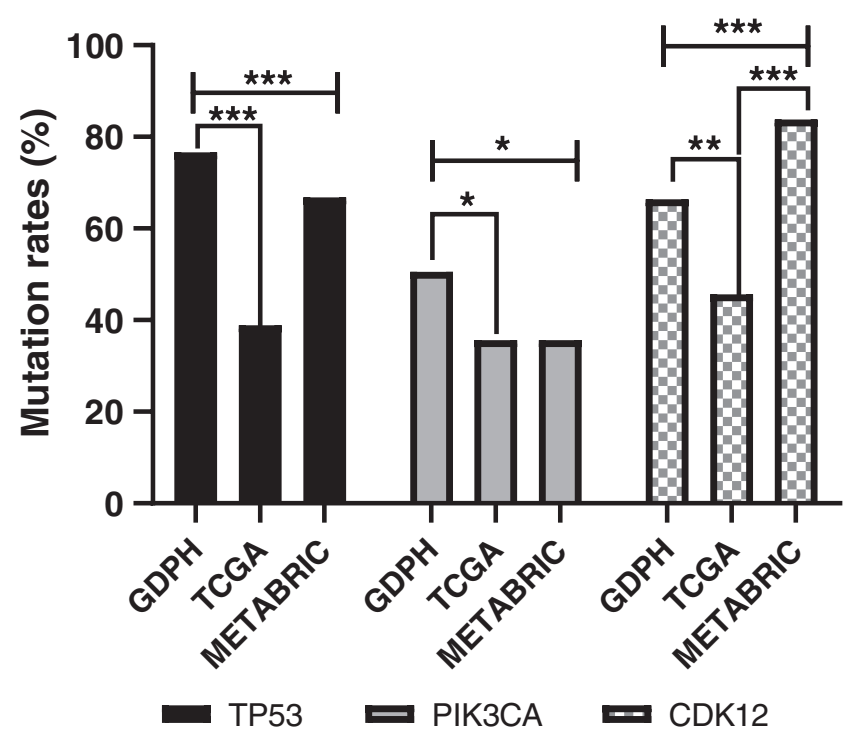

Figure 4

The mutation rates of TP53, PIK3CA and CDK12 in HER2+ breast cancer. We compared the data from GDPH with the HER2+ breast cancer dataset from The Cancer Genome Atlas (TCGA; $n=90$ ) and Molecular Taxonomy of Breast Cancer International Consortium (METABRIC; $n=247)$. * indicated $P$-values $<0.05, * *$ indicated $P$-values $<0.01$ and $* * *$ indicated $P$-values $<0.001$. A full colour version of this figure is available at https:// doi.org/10.1530/ERC-19-0414. 
Table 2 Aberrant events of the ERBB family in HER2-positive breast cancer.

\begin{tabular}{|c|c|c|c|c|}
\hline & EGFR & HER2 & HER3 & HER4 \\
\hline HR+/HER2+ & c. $1631+5 G>C$ & $\begin{array}{l}\text { Fusion (KRTAP1-4-ERBB2) } \\
\text { Fusion (METRNL-ERBB2) } \\
\text { Fusion (PIP4K2B-ERBB2) } \\
\text { Fusion (MED1-ERBB2) } \\
\text { p.D769Y } \\
\text { p.L869R } \\
\text { p.R217C }\end{array}$ & $\begin{array}{l}\text { p.E928G } \\
\text { p.N522S } \\
\text { p.Q607K }\end{array}$ & p.V395L \\
\hline HR-/HER2+ & $\begin{array}{l}\text { cn_amp }(n=2) \\
\text { p.A289T }\end{array}$ & $\begin{array}{l}\text { Fusion (SRCIN1-ERBB2) } \\
\text { Fusion (GLRA3-ERBB2) } \\
\text { p.D769Y } \\
\text { p.V777L }\end{array}$ & & \\
\hline
\end{tabular}

we identified 17 HER2-positive breast cancer patients (15.9\%, 17/107) with 19 concurrent mutations in other members of the ERBB gene family. Among the members of the ERBB family, ERBB2 had the most variants detected, with a total of 11 gene mutations including five missense mutations and six fusions detected in the cohort. In addition, our data revealed the detection of two novel missense mutations in HER2: L869R and R217C, in an HR+/HER2+ patient each, which were not previously reported or found in databases such as TCGA (Cancer Genome Atlas Network 2012) and METABRIC (Curtis et al. 2012). Interestingly, two of the most commonly observed mutations in the abovementioned public databases, HER2 P523S and L755S, were not observed from our cohort. Instead, HER2 D769Y and V777L, which were also reported in public databases, were detected from our cohort. HER2 D769Y was detected from a patient with HR+/HER2+ tumor, while HER2 D769Y and V777L were each detected from two patients with HR-/HER2+ tumor. EGFR mutations were detected from four $(4.1 \%)$ tumors, including one missense mutation (A289T), one splice region variant (c.1631+5G>C) and two $\mathrm{CN}$ amplifications. Interestingly, ERBB3 and $E R B B 4$ missense mutations were only detected in HR+/HER2+ tumors. Of which, three previously unreported missense mutations in ERBB3, E928G, Q607K and N522S, were detected from three (4.7\%, 3/64) HR+/HER2+ patients. Meanwhile, a novel missense ERBB4 V395L was detected from an HR+/HER2+ patient. In addition to HER2 amplifications, concurrent fusions in ERBB2 $(67 \%, 4 / 6)$ and SNVs in ERBB3 $(100 \%$, $3 / 3)$ and $E R B B 4(100 \%, 1 / 1)$ were more frequently detected among HR+/HER2+ tumors, while concurrent EGFR CN amplifications $(2 / 2)$ were exclusively detected among HR-/HER2+ tumors.

\section{Discussion}

HER2-positive breast cancer is a clinically distinct class of breast cancers for which several targeted therapies are now

(c) 2020 Society for Endocrinology Published by Bioscientifica Ltd. Printed in Great Britain available. Here, we have identified somatic mutations in HER2-positive breast cancers and discussed the potential association between clinical treatment efficacy and tumor mutation landscape. Our study demonstrated that HER2positive breast tumors have more frequent mutations in TP53, CDK12 and PIK3CA, with mutation rates of 76, 61 and $48 \%$, respectively. The mutations in TP53 and PIK3CA were mainly single nucleotide variations, while CDK12 was mainly gene amplification. This finding is consistent with previous studies showing that mutations in TP53 and PIK3CA were commonly detected in breast tumors and may play a very important role in the progression of breast cancer (Powell et al. 2014, Lefebvre et al. 2016, Schrijver et al. 2018). In contrast to previously reported study, the HER2-positive breast cancer patients included in our cohort had significantly more TP53 mutations (76\%, $81 / 107$ vs $44 \%, 28 / 64$ ) (Ferrari et al. 2016). Consistently, as compared to the TCGA and METABRIC dataset, our cohort had significantly higher mutation rates of TP53, PIK3CA and CDK12 than TCGA, while our cohort had a significantly higher PIK3CA mutation rate, a trend of higher TP53 mutation rate and significantly lower CDK12 mutation rate as compared to the METABRIC dataset. Our results strongly indicate that Chinese HER2-positive breast cancers patients had distinct genomic alterations which might lead to different biological characteristics and potentially affect their treatment responses and clinical outcomes. CDK12 is another frequently mutated gene from our cohort. Genomic alterations in CDK12 have been detected in various cancer types including stomach, esophageal, endometrial, uterine, ovarian, bladder, colorectal and pancreatic cancers, with mutation detection rate ranging from 5\% to 15\% (Lui et al. 2018). The CDK12 is located in chromosome 17 (17q12), approximately $200 \mathrm{~kb}$ proximal to the HER2 oncogene (Kauraniemi \& Kallioniemi 2006) and it is frequently co-amplified with HER2 in breast cancer (Tien et al. 2017). Consistently, we have also detected a high frequency of 
CDK12 amplification from our cohort of HER2-positive breast cancer patients. CDK12 is a transcriptional cyclindependent kinase (CDK) that complexes with cyclin $\mathrm{K}$ to mediate gene transcription (Bartkowiak et al. 2010). It also has been demonstrated to specifically upregulate the expression of genes involved in response to DNA damage, heat shock and stress. CDK12 gene amplification could contribute to cancer fitness by constitutive engagement of DNA repair pathways (Lui et al. 2018). CDK12 inhibition has been explored as an effective strategy to repress tumor growth, and synthetic lethal interactions have been described with PARP inhibition and CDK12 as a clinical biomarker for response in cancer treatment (Bajrami et al. 2014, Johnson et al. 2016). Further study of the role of CDK12 in HER2-positive breast cancers will help us better understand the biological characteristics of HER2-positive breast cancers and discover potential treatments for patients with concomitant CDK12 amplifications.

When analyzing the genomic difference between HR+/HER2+ and HR-/HER2+, it was apparent that HR+/HER2+ had more gene amplification than HR-/HER2+ patients. Concurrent gene amplifications in $S P O P$, CCND1, FGF19, FGF4, FGF3, RNF43, RAD51C, ADGRA2 and MDM4 and various mutations in GATA3 were more frequently detected in HR+/HER2+ tumors as compared to HR-/HER2+ tumors. Among all the mutation types, gene amplifications were also significantly more in $\mathrm{HR}+/ \mathrm{HER} 2+$ than HR-/HER2+ patients in the METABRIC dataset. The frequency of copy number amplifications in our cohort was comparable with that of the TCGA dataset and was fewer than in the METABRIC dataset. To further explore a potential clinical relevance of this finding, we analyzed the correlation between the distribution of copy number amplification and clinical features in HR+/HER2+ and HR-/HER2+ groups. There was no significant difference in the Ki67 expression levels between HR+/HER2+ and HR-/HER2+ groups $(P=0.237)$. Interestingly, HR+/HER2+ patients with copy number amplifications, particularly in chromosomal regions that involved $C D 79 B(P=0.037)$, PRKAR1A $(P=0.035)$ or $R A D 51 C(P=0.047)$, had significantly higher expression of Ki67 than HR-/HER2+ patients. Ki67 antigen is routinely used as a marker of tumor cell proliferation and growth and is associated with treatment responses and survival outcomes (Li et al. 2015). We speculate that the concurrent copy number amplification may contribute to the treatment refractory characteristics of HR+/HER2+ patients. However, analysis with a larger cohort with relevant clinical data including treatment information and survival outcomes are required to further explore this association. Recently, therapeutic agents that target mutations in CCND1, FGF19, FGF4 and FGF3 have been developed. Therapeutic strategies involving combination therapies might potentially improve the prognosis of the subset of HER2-positive breast patients with concurrent mutations in these genes. Moreover, patients with HR+/HER2+ have significant differences with HR-/HER2+ patients in TGF-beta $(P=0.007)$, WNT $(P=0.002)$, and HRR $(P=0.004)$ signaling pathways. At present, there are many therapeutic agents that are designed to target the HRR pathway, such as PARP inhibitors, that could serve as treatment options for this subset of patients (Papadimitriou et al. 2018). Our results suggest the potential application of PARP inhibitors for HR+/HER2+ patients with concurrent mutations in genes involved in the HRR pathway.

HER2 belongs to the ERBB receptor family, which serves as an excellent candidate for therapeutic intervention based on studies of tumor formation (Saxena \& Dwivedi 2012, Arteaga \& Engelman 2014). The ERBB family members promote tumor cell proliferation as well as survival in a variety of malignancies including breast cancer (Saxena \& Dwivedi 2012). In our present study, we also identified several novel mutations that were previously unreported in breast cancer databases. In addition to HER2 amplifications, concurrent fusions in ERBB2 $(67 \%, 4 / 6)$ and SNVs in ERBB3 $(100 \%, 3 / 3)$ and ERBB4 (100\%, 1/1) were more likely to be detected from HR+/HER2+ tumors, while concurrent EGFR amplifications were exclusively detected from HR-/HER2+ tumors. The oncogenic potential and clinical implications of these novel mutations concurrent to HER2 amplifications are still unknown and warrant further investigations.

Despite our interesting findings, our study is limited by the lack of data for clinical outcomes of the HER2positive breast cancer patients with various concurrent mutations. Our study also did not include an analysis of the germline genomic profile of our cohort, nor any functional analysis on the concurrent mutations detected from our cohort. A multi-center prospective study with a larger cohort is necessary to investigate and extend the clinical relevance of our findings.

\section{Conclusion}

In conclusion, we analyzed the somatic mutation characteristics of HER2-positive breast cancer. We found that Chinese HER2-positive breast cancer patients have a distinct somatic mutation profile than Caucasian patients, 
with higher mutation rate of TP53, CDK12 and PIK3CA and potentially involves different signaling pathways. Moreover, concurrent gene amplification is distinct in HR+/HER2-positive breast cancer. Our study suggests that the intrinsic genomic characteristics of HER2-positive breast cancer adds a layer of biological complexity, worthy of further investigation for tailoring better therapeutic strategies to improve the prognosis of this subset of patients.

\section{Supplementary materials}

This is linked to the online version of the paper at https://doi.org/10.1530/ ERC-19-0414.

\section{Declaration of interest}

Ting Hou, Han Han-Zhang and Jing Liu are employees of Burning Rock Biotech. The other authors have nothing to declare.

\section{Funding}

This work was supported by funds from High-level Hospital Construction Project (DFJH201921, Bo Chen); Natural Science Foundation of Guangdong Province (2016A030313768, Ning Liao; 2018A030313292) and Research Funds from Guangzhou Municipal Science and Technology Project (201707010418, Ning Liao); the National Natural Science Foundation of China (81902828, Bo Chen; 81602645, Yulei Wang); the Fundamental Research Funds for the Central Universities (y2syD2192230, Bo Chen); Medical Scientific Research Foundation of Guangdong Province (B2019039, Bo Chen) and Research Funds from Guangzhou Municipal Science and Technology Project (grant numbers: 201707010418; 201804010430). The funding bodies have no role in the design of the study, the collection, analysis and interpretation of data and in writing the manuscript.

\section{Ethics approval and consent to participate}

Primary tumor biopsies were obtained from the patients using an Institutional Review Board-approved protocol, and this study had been approved by the Ethic Committee of Guangdong Provincial People's Hospital. All the patients provided written informed consent for translational research.

\section{Availability of supporting data}

The datasets generated and analyzed during the current study are in the process of being deposited to a public databank. Accession number will be provided once all the data are completely uploaded.

\section{Author contribution statement}

N L, B C and G Z designed the study. B C, G Z, G W, Y W, L G, L C, C R, $L W, K L, M J, C L, H M$ and J $L$ participated in sample collection, sample processing, clinical information collection and data analysis. N L, B C and C B participated in the analysis, interpretation of the data and manuscript writing. All the authors read and approved the final manuscript.

\section{Acknowledgements}

The authors thank all the patients and their families for participation.

\section{References}

Arteaga CL \& Engelman JA 2014 ERBB receptors: from oncogene discovery to basic science to mechanism-based cancer therapeutics. Cancer Cell 25 282-303. (https://doi.org/10.1016/j.ccr.2014.02.025)

Bajrami I, Frankum JR, Konde A, Miller RE, Rehman FL, Brough R, Campbell J, Sims D, Rafiq R, Hooper S, et al. 2014 Genome-wide profiling of genetic synthetic lethality identifies CDK12 as a novel determinant of PARP1/2 inhibitor sensitivity. Cancer Research $\mathbf{7 4}$ 287-297. (https://doi.org/10.1158/0008-5472.CAN-13-2541)

Bartkowiak B, Liu P, Phatnani HP, Fuda NJ, Cooper JJ, Price DH, Adelman K, Lis JT \& Greenleaf AL 2010 CDK12 is a transcription elongation-associated CTD kinase, the metazoan ortholog of yeast Ctk1. Genes and Development 24 2303-2316. (https://doi.org/10.1101/ gad.1968210)

Cancer Genome Atlas Network 2012 Comprehensive molecular portraits of human breast tumours. Nature 490 61-70. (https://doi. org/10.1038/nature11412)

Cortazar P, Zhang L, Untch M, Mehta K, Costantino JP, Wolmark N, Bonnefoi H, Cameron D, Gianni L, Valagussa P, et al. 2014 Pathological complete response and long-term clinical benefit in breast cancer: the CTNeoBC pooled analysis. Lancet 384 164-172. (https://doi.org/10.1016/S0140-6736(13)62422-8)

Curtis C, Shah SP, Chin SF, Turashvili G, Rueda OM, Dunning MJ, Speed D, Lynch AG, Samarajiwa S, Yuan Y, et al. 2012 The genomic and transcriptomic architecture of 2,000 breast tumours reveals novel subgroups. Nature 486 346-352. (https://doi.org/10.1038/ nature10983)

DeSantis CE, Ma J, Goding Sauer A, Newman LA \& Jemal A 2017 Breast cancer statistics, 2017, racial disparity in mortality by state. CA: A Cancer Journal for Clinicians 67 439-448. (https://doi.org/10.3322/ caac.21412)

Ferrari A, Vincent-Salomon A, Pivot X, Sertier AS, Thomas E, Tonon L, Boyault S, Mulugeta E, Treilleux I, Macgrogan G, et al. 2016 A whole-genome sequence and transcriptome perspective on HER2positive breast cancers. Nature Communications 7 12222. (https://doi. org/10.1038/ncomms12222)

Gingras I, Gebhart G, De Azambuja E \& Piccart-Gebhart M 2017 HER2positive breast cancer is lost in translation: time for patient-centered research. Nature Reviews: Clinical Oncology 14 669-681. (https://doi. org/10.1038/nrclinonc.2017.96)

Hyman DM, Piha-Paul SA, Won H, Rodon J, Saura C, Shapiro GI, Juric D, Quinn DI, Moreno V, Doger B, et al. 2018 HER kinase inhibition in patients with HER2- and HER3-mutant cancers. Nature 554 189-194. (https://doi.org/10.1038/nature25475)

Jaiswal BS, Kljavin NM, Stawiski EW, Chan E, Parikh C, Durinck S, Chaudhuri S, Pujara K, Guillory J, Edgar KA, et al. 2013 Oncogenic ERBB3 mutations in human cancers. Cancer Cell 23 603-617. (https://doi.org/10.1016/j.ccr.2013.04.012)

Johnson SF, Cruz C, Greifenberg AK, Dust S, Stover DG, Chi D, Primack B, Cao S, Bernhardy AJ, Coulson R, et al. 2016 CDK12 inhibition reverses de novo and acquired PARP inhibitor resistance in BRCA wild-type and mutated models of triple-negative breast cancer. Cell Reports 17 2367-2381. (https://doi.org/10.1016/j. celrep.2016.10.077)

Kauraniemi P \& Kallioniemi A 2006 Activation of multiple cancerassociated genes at the ERBB2 amplicon in breast cancer. EndocrineRelated Cancer 13 39-49. (https://doi.org/10.1677/erc.1.01147)

Lefebvre C, Bachelot T, Filleron T, Pedrero M, Campone M, Soria JC, Massard C, Levy C, Arnedos M, Lacroix-Triki M, et al. 2016 Mutational profile of metastatic breast cancers: a retrospective analysis. PLoS Medicine 13 e1002201. (https://doi.org/10.1371/ journal.pmed.1002201)

Li LT, Jiang G, Chen Q \& Zheng JN 2015 Ki67 is a promising molecular target in the diagnosis of cancer (review). Molecular Medicine Reports 11 1566-1572. (https://doi.org/10.3892/mmr.2014.2914) https://erc.bioscientifica.com

https://doi.org/10.1530/ERC-19-0414 (c) 2020 Society for Endocrinology Published by Bioscientifica Ltd. Printed in Great Britain 
Loibl S, Von Minckwitz G, Schneeweiss A, Paepke S, Lehmann A, Rezai M, Zahm DM, Sinn P, Khandan F, Eidtmann H, et al. 2014 PIK3CA mutations are associated with lower rates of pathologic complete response to anti-human epidermal growth factor receptor 2 (her2) therapy in primary HER2-overexpressing breast cancer. Journal of Clinical Oncology 32 3212-3220. (https://doi.org/10.1200/ JCO.2014.55.7876)

Lui GYL, Grandori C \& Kemp CJ 2018 CDK12: an emerging therapeutic target for cancer. Journal of Clinical Pathology 71 957-962. (https:// doi.org/10.1136/jclinpath-2018-205356)

Ng CK, Martelotto LG, Gauthier A, Wen HC, Piscuoglio S, Lim RS, Cowell CF, Wilkerson PM, Wai P, Rodrigues DN, et al. 2015 Intratumor genetic heterogeneity and alternative driver genetic alterations in breast cancers with heterogeneous HER2 gene amplification. Genome Biology 16 107. (https://doi.org/10.1186/ s13059-015-0657-6)

Papadimitriou M, Mountzios G \& Papadimitriou CA 2018 The role of PARP inhibition in triple-negative breast cancer: unraveling the wide spectrum of synthetic lethality. Cancer Treatment Reviews 67 34-44. (https://doi.org/10.1016/j.ctrv.2018.04.010)

Piccart-Gebhart M, Holmes E, Baselga J, De Azambuja E, Dueck AC, Viale G, Zujewski JA, Goldhirsch A, Armour A, Pritchard KI, et al. 2016 Adjuvant lapatinib and trastuzumab for early human epidermal growth factor receptor 2-positive breast cancer: results from the randomized phase III adjuvant lapatinib and/or trastuzumab treatment optimization trial. Journal of Clinical Oncology 34 1034-1042. (https://doi.org/10.1200/ JCO.2015.62.1797)

Pitt JJ, Riester M, Zheng Y, Yoshimatsu TF, Sanni A, Oluwasola O, Veloso A, Labrot E, Wang S, Odetunde A, et al. 2018 Characterization of Nigerian breast cancer reveals prevalent homologous recombination deficiency and aggressive molecular features. Nature Communications 9 4181. (https://doi.org/10.1038/ s41467-018-06616-0)
Powell E, Piwnica-Worms D \& Piwnica-Worms H 2014 Contribution of p53 to metastasis. Cancer Discovery 4 405-414. (https://doi. org/10.1158/2159-8290.CD-13-0136)

Saxena R \& Dwivedi A 2012 ErbB family receptor inhibitors as therapeutic agents in breast cancer: current status and future clinical perspective. Medicinal Research Reviews 32 166-215. (https://doi. org/10.1002/med.20209)

Schrijver WAME, Selenica P, Lee JY, Ng CKY, Burke KA, Piscuoglio S, Berman SH, Reis-Filho JS, Weigelt B, van Diest PJ, et al. 2018 Mutation profiling of key cancer genes in primary breast cancers and their distant metastases. Cancer Research 78 3112-3121. (https://doi. org/10.1158/0008-5472.CAN-17-2310)

Tien JF, Mazloomian A, Cheng SG, Hughes CS, Chow CCT, Canapi LT, Oloumi A, Trigo-Gonzalez G, Bashashati A, Xu J, et al. 2017 CDK12 regulates alternative last exon mRNA splicing and promotes breast cancer cell invasion. Nucleic Acids Research 45 6698-6716. (https:// doi.org/10.1093/nar/gkx187)

Toomey S, Eustace AJ, Fay J, Sheehan KM, Carr A, Milewska M, Madden SF, Teiserskiene A, Kay EW, O'Donovan N, et al. 2017 Impact of somatic PI3K pathway and ERBB family mutations on pathological complete response (pCR) in HER2-positive breast cancer patients who received neoadjuvant HER2-targeted therapies. Breast Cancer Research 19 87. (https://doi.org/10.1186/s13058-017-0883-9)

Von Minckwitz G, Untch M, Blohmer JU, Costa SD, Eidtmann H, Fasching PA, Gerber B, Eiermann W, Hilfrich J, Huober J, et al. 2012 Definition and impact of pathologic complete response on prognosis after neoadjuvant chemotherapy in various intrinsic breast cancer subtypes. Journal of Clinical Oncology 30 1796-1804. (https://doi. org/10.1200/JCO.2011.38.8595)

Zhang G, Wang Y, Chen B, Guo L, Cao L, Ren C, Wen L, Li K, Jia M, Li C, et al. 2019 Characterization of frequently mutated cancer genes in Chinese breast tumors: a comparison of Chinese and TCGA cohorts. Annals of Translational Medicine 7 179. (https://doi. org/10.21037/atm.2019.04.23)

Received in final form 22 November 2019

Accepted 24 December 2019

Accepted Manuscript published online 6 January 2020 (c) 2020 Society for Endocrinology Published by Bioscientifica Ltd. Printed in Great Britain 